\title{
Aspirin therapy in patients with acute respiratory distress syndrome (ARDS) is associated with reduced intensive care unit mortality: a prospective analysis
}

\author{
Andrew J Boyle ${ }^{1,2^{*}}$, Stefania Di Gangi ${ }^{3}$, Umar I Hamid', Linda-Jayne Mottram², Lia McNamee², Griania White²,
} LJ Mark Cross ${ }^{1,2}$, James J McNamee ${ }^{2}$, Cecilia M O'Kane ${ }^{1}$ and Daniel F McAuley ${ }^{1,2}$

\begin{abstract}
Introduction: Acute respiratory distress syndrome (ARDS) is a common clinical syndrome with high mortality and long-term morbidity. To date there is no effective pharmacological therapy. Aspirin therapy has recently been shown to reduce the risk of developing ARDS, but the effect of aspirin on established ARDS is unknown.

Methods: In a single large regional medical and surgical ICU between December 2010 and July 2012, all patients with ARDS were prospectively identified and demographic, clinical, and laboratory variables were recorded retrospectively. Aspirin usage, both pre-hospital and during intensive care unit (ICU) stay, was included. The primary outcome was ICU mortality. We used univariate and multivariate logistic regression analyses to assess the impact of these variables on ICU mortality.

Results: In total, 202 patients with ARDS were included; 56 (28\%) of these received aspirin either pre-hospital, in the ICU, or both. Using multivariate logistic regression analysis, aspirin therapy, given either before or during hospital stay, was associated with a reduction in ICU mortality (odds ratio (OR) $0.38(0.15$ to 0.96$) P=0.04$ ). Additional factors that predicted ICU mortality for patients with ARDS were vasopressor use (OR 2.09 (1.05 to 4.18) $P=0.04)$ and APACHE II score (OR 1.07 (1.02 to 1.13) $P=0.01)$. There was no effect upon ICU length of stay or hospital mortality.
\end{abstract}

Conclusion: Aspirin therapy was associated with a reduced risk of ICU mortality. These data are the first to demonstrate a potential protective role for aspirin in patients with ARDS. Clinical trials to evaluate the role of aspirin as a pharmacological intervention for ARDS are needed.

\section{Introduction}

Acute respiratory distress syndrome (ARDS) is a common devastating clinical syndrome characterised by life-threatening hypoxaemic respiratory failure often requiring mechanical ventilation and frequently leading to multiple organ failure. ARDS is a major cause of morbidity and mortality within the ICU, and causes long-term reduction in quality of life for survivors [1-3].

\footnotetext{
* Correspondence: boyle.andrewj@gmail.com

${ }^{1}$ Centre for Infection and Immunity, Health Sciences Building, Queen's University Belfast, 97 Lisburn Road, Belfast, UK

${ }^{2}$ Regional Intensive Care Unit, Royal Victoria Hospital, Grosvenor Road, Belfast, UK

Full list of author information is available at the end of the article
}

ARDS is an inflammatory condition characterised by neutrophil-mediated $[4,5]$ and macrophage-mediated [6] injury. This uncontrolled local inflammatory response causes alveolar epithelial and capillary endothelial barrier damage, increasing its permeability. This allows the accumulation of an inflammatory infiltrate, and proteinaceous fluid within the alveolar space (non-cardiogenic pulmonary oedema) that contributes to profound hypoxaemia. The accompanying widespread activation of the coagulation cascade leads to microvascular thrombosis and fibroproliferation [7]. Currently there are few effective interventions for ARDS [8,9], and these primarily involve limiting ventilator-induced lung injury with low tidal volume 
ventilation [10], prone positioning [11], with emerging data for neuromuscular blockade [12] and extra-corporeal therapies [13,14].

Platelets have an increasingly recognised role in the inflammatory response leading to the development of ARDS. Platelet activation mediates neutrophil-recruitment to the lung in an acid-induced murine model of lung injury, an effect that is inhibited by pre-treatment with aspirin [15]. Platelet depletion in two mouse models of ARDS reduced the severity of lung injury and increased survival, an effect that was reproduced by pre-treatment with aspirin [16]. In addition, delayed neutrophil apoptosis is a feature of ARDS that aspirin can ameliorate to promote resolution of persisting inflammation [17].

Most, but not all $[18,19]$ observational data suggest that aspirin use prior to ICU admission (without ARDS at the point of admission) may reduce the subsequent development of ARDS [20-22]. Finally, in a cohort of patients admitted with community-acquired pneumonia, those patients being treated with anti-platelet drugs (the majority of whom received aspirin) had a significantly lower rate of critical care admission [23].

These data suggest that aspirin may prevent ARDS, however, it is unknown if aspirin exposure alters outcome in patients with established ARDS. Aspirin exposure in patients with systemic inflammatory response syndrome (SIRS), severe sepsis or septic shock is associated with reduced mortality [24,25], but there are no data in patients with ARDS. We hypothesised that aspirin treatment, either prior to, or during ICU admission, would reduce mortality in patients with ARDS. To assess this, we prospectively identified patients with ARDS to determine the effect of aspirin exposure on mortality within ICU.

\section{Methods}

We conducted an audit of ARDS management between December 2010 and July 2012. This was a convenience sample and a formal sample size calculation was not performed. The Belfast Health and Social Care Trust determined this project as an audit, because patient management was not altered, only routinely collected data were used and the data were fully anonymised, and as a result research ethics committee approval was not required. The requirement for patient consent was therefore waived.

\section{Patient population}

All adult patients ( $>16$ years-old) requiring invasive mechanical ventilation admitted to the 17-bed mixed medical and surgical Regional Intensive Care Unit in the Royal Victoria Hospital, Belfast, were prospectively screened daily as part of ongoing clinical trials of ARDS (53 of the patients included in this analysis were randomised to a clinical trial). Patients who met the North
American-European consensus conference definition of acute lung injury [26] (a term now replaced by ARDS [27], and to which we refer throughout) were identified prospectively and included. There were no exclusion criteria. Determination of ARDS was made by trained research coordinators who are involved in the screening of patients with ARDS for recruitment into clinical trials ongoing within the ICU, and where there was any uncertainty the attending physician confirmed the diagnosis of ARDS. Patients were screened daily from admission for the diagnosis of ARDS, and were included in this cohort once they met the criteria for diagnosis.

\section{Data collection}

Baseline demographics recorded were age, gender, and acute physiology and chronic health evaluation (APACHE) II score at admission to ICU. We recorded a medical history of alcohol dependency, smoking, liver cirrhosis, ischaemic heart disease, congestive heart failure, chronic obstructive airways disease, cerebrovascular disease and diabetes mellitus. We also recorded whether patients received a statin prior to admission. Medication history was obtained through chart review and medication reconciliation completed by the ICU pharmacist, who contacted the community general practitioner to complete these data if necessary. The ratio of partial pressure of arterial oxygen $\left(\mathrm{PaO}_{2}\right)$ to fraction of inspired oxygen $\left(\mathrm{FiO}_{2}\right)$, sequential organ failure assessment (SOFA) score on the day of ARDS diagnosis and the aetiology of ARDS was recorded. Aspirin usage both pre-admission and during ICU stay was included. Patients were deemed to have been treated with aspirin if it was part of their prehospital medication, or if given whilst in ICU (or both). All records were reviewed by a qualified physician.

\section{Primary and secondary outcomes}

The primary outcome was ICU mortality. Secondary outcomes included duration of ICU stay and hospital mortality.

\section{Statistical methods}

We compared patients with ARDS who received aspirin (aspirin ever) with those who did not (no aspirin). Baseline characteristics and outcome variables were compared using standard tests for continuous and binary variables. We reported results of the Kruskal-Wallis or chi squared test, as appropriate, when we compared three or more groups.

Data are presented as mean (SD), median (IQR) and number (\%) as appropriate. The number of non-missing observations is shown when different from the overall population. The distributions of all variables were tested for normality. The independent sample Student's $t$-test was used for continuous variables with a normal distribution. 
Otherwise, the Wilcoxon-Mann-Whitney was used when normality was violated. The chi squared test or Fisher's exact two-sided test, as appropriate, were used for binary variables. A $P$-value $\leq 0.05$ was considered statistically significant.

Univariate and multivariate logistic regression models were performed to identify the association between baseline characteristics and the primary outcome. We used multivariate logistic regressions to calculate (adjusted) odds ratios (OR) and 95\% confidence intervals (CI) for the association between aspirin use and outcomes. Adjustments for any potential confounding effects were made for clinically relevant variables and for those that showed a statistically significant difference between the groups at baseline.

We further examined the adjusted effect of aspirin use on the primary and secondary outcomes with multivariate Cox proportional hazards regression models. The dates of admission to ICU or hospital, respectively, were the starting points of the survival analyses and the dates of discharge from ICU, or hospital, were the endpoints.

Multivariate models were constructed using automatic stepwise selection estimation with likelihood ratio testing $(P$-value $\leq 0.20)$ specified as the test of significance to include or exclude variables. Aspirin exposure (as the variable of interest), and history of coronary artery disease (as a significant, potential confounder) were locked into the model and not subject to the selection criteria. In a secondary analysis, combined aspirin and statin exposure, and history of coronary artery disease, were locked into the model. APACHE and SOFA as severity of illness scores were not both included in the multivariate model because of the problem of co-linearity in that the scores are correlated, because the severity of illness variables by which they are calculated are broadly the same or correlated. The Kaplan-Meier curve was used to plot ICU survival between patient groups with the log rank test used to determine differences among these groups. STATA SE version 11.0 (StataCorp LP, TX, USA) was used for all analyses. Graphs were constructed using GraphPad Prism (v 6.0).

\section{Results}

Two hundred and two patients with ARDS were included; 56 (28\%) received aspirin either pre-hospital ( $\mathrm{n}=31,55 \%$ of total aspirin), in ICU ( $\mathrm{n}=7,13 \%)$ or both pre-hospital and in ICU ( $\mathrm{n}=18,32 \%)$. The dose of aspirin received ranged from $75 \mathrm{mg}$ daily $(\mathrm{n}=53,95 \%)$ to $300 \mathrm{mg}$ daily. Patient demographics are summarised in Table 1. Patients treated with aspirin were likely to be older (71 versus $56 ; P<0.001$ ) and have greater comorbidities. The use of statin therapy was higher in the aspirin group (37 versus $24 ; P=<0.001$ ).

\section{ICU mortality}

The primary analysis involved those who had taken aspirin ever compared with those never exposed. In univariate analysis four variables were associated with ICU mortality; patient age, APACHE II score, SOFA score and vasopressor use (Table 2). Although aspirin exposure was not associated with an impact upon mortality in the univariate analysis (Table 2), in a multiple logistic regression analysis, treatment with aspirin was associated with a significantly lower ICU mortality compared with patients who had no aspirin exposure (OR 0.38 (0.15 to $0.96) ; P=0.04)$. The use of vasopressors during admission (OR 2.09 (1.05 to 4.18); $P=0.04$ ) and APACHE II score at admission (OR 1.07 (1.02 to 1.13 ); $P=0.01$ ) was associated with an increased risk of death (Table 3).

In analysis of the combined effect of aspirin and statin therapy, patients exposed to both drugs had no ICU survival advantage (OR $0.68(0.26,1.80)$; $P=0.44)$ (Table 4).

To determine the effect of timing of treatment we analysed the different subgroups of patients treated with aspirin (aspirin pre-ICU, aspirin pre-ICU and in ICU, aspirin in ICU only) and those never treated with aspirin. Although there was increased survival in patients started on aspirin within ICU, there was no significant difference in survival amongst the groups (log rank test, $P=0.48$ ) (Figure 1 ).

\section{Length of ICU stay}

Aspirin treatment was not associated with a significant impact upon ICU length of stay (hazard ratio (HR) 0.64 (0.33 to 1.25), $\mathrm{P}=0.19$ ), whilst APACHE II score correlated with a longer ICU admission (HR 1.07 (1.03 to 1.12), $\mathrm{P}=0.001) P=0.001$ ).

\section{Hospital mortality}

The association of aspirin did not extend to overall hospital mortality (OR 0.91 (0.46 to 1.78 ); $P=0.78$ ). APACHE II score positively correlated with hospital mortality (OR 1.09 (1.04 to 1.14 ); $P<0.001$ ).

\section{Discussion}

In this prospectively identified audit of ARDS management, we found aspirin therapy to be associated with a reduced risk of ICU mortality from ARDS. Using multivariate analysis in patients with ARDS, we found aspirin was an independent factor associated with a reduction in risk of death within ICU, whilst the use of vasopressors and APACHE II score at ICU admission correlated with increased risk of ICU death. This is the first report to find aspirin therapy is associated with reduced ICU mortality in patients with established ARDS.

There are several processes that aspirin may modulate to provide a beneficial effect in ARDS. Aspirin has significant anti-platelet properties through the inhibition 
Table 1 Baseline characteristics of patients with acute lung injury

\begin{tabular}{|c|c|c|c|c|}
\hline & $\begin{array}{l}\text { Overall } \\
(n=202)\end{array}$ & $\begin{array}{l}\text { Aspirin ever } \\
(\mathrm{n}=56,28 \%)\end{array}$ & $\begin{array}{l}\text { No aspirin } \\
(n=146,72 \%)\end{array}$ & $P$-value \\
\hline \multicolumn{5}{|l|}{ Age } \\
\hline Median & 61 & 71 & 56 & $<0.001^{*}$ \\
\hline IQR & 46 to 71 & 60 to 77 & 41 to 68 & \\
\hline \multicolumn{5}{|l|}{ Male } \\
\hline Number (\%) & $129(64)$ & $40(71)$ & $89(61)$ & 0.17 \\
\hline \multicolumn{5}{|l|}{ APACHE I| score } \\
\hline Median & 19 & 21 & 18 & 0.10 \\
\hline IQR & 14 to 24 & 17 to 24 & 13 to 24 & \\
\hline \multicolumn{5}{|l|}{ SOFA } \\
\hline Median & 8 & 8 & 8 & 0.99 \\
\hline IQR & 6 to 11 & 7 to 11 & 6 to 11 & \\
\hline \multicolumn{5}{|l|}{$\mathrm{PaO}_{2} / \mathrm{FiO}_{2}$ ratio } \\
\hline Median & 22.9 & 21.1 & 23.5 & 0.08 \\
\hline IQR & 17 to 30 & 16 to 27 & 18 to 31 & \\
\hline \multicolumn{5}{|l|}{ Vasopressor use } \\
\hline Number (\%) & $95(47)$ & $30(54)$ & $65(45)$ & 0.25 \\
\hline \multicolumn{5}{|l|}{ Sepsis } \\
\hline Number (\%) & $111(55)$ & $29(52)$ & $82(56)$ & 0.58 \\
\hline \multicolumn{5}{|c|}{ Coronary artery disease } \\
\hline N (\%) & $33(16)$ & $22(39)$ & $11(8)$ & $<0.001$ \\
\hline \multicolumn{5}{|c|}{ Cerebrovascular disease } \\
\hline Number (\%) & $12(6)$ & $9(16)$ & $3(2)$ & $0.001 *$ \\
\hline \multicolumn{5}{|l|}{ COPD } \\
\hline Number (\%) & $29(14)$ & $13(23)$ & $16(11)$ & $0.02 *$ \\
\hline \multicolumn{5}{|l|}{ Diabetes mellitus } \\
\hline Number (\%) & $30(15)$ & $18(32)$ & $12(8)$ & $<0.001^{*}$ \\
\hline \multicolumn{5}{|l|}{ Smoker } \\
\hline Number (\%) & $99(49)$ & $34(61)$ & $65(45)$ & $0.04 *$ \\
\hline \multicolumn{5}{|c|}{ Alcohol abuse/liver cirrhosis } \\
\hline Number (\%) & $58(29)$ & $9(16)$ & $49(34)$ & $0.01 *$ \\
\hline \multicolumn{5}{|c|}{ Pre-admission statin } \\
\hline Number (\%) & $61(30)$ & $37(66)$ & $24(16)$ & $<0.001^{*}$ \\
\hline
\end{tabular}

*Values that were deemed significant. APACHE II, acute physiology and chronic health evaluation; SOFA, sequential organ failure assessment; PaO ${ }_{2}$, arterial oxygen partial pressure; $\mathrm{FiO}_{2}$, fraction of inspired oxygen; $\mathrm{COPD}$, chronic obstructive pulmonary disease.

of cyclo-oxygenase enzymes that prevent thromboxane A2 production, therefore suppressing platelet aggregation; an effect that can last up to 10 days post administration [28]. Platelets aggregate at sites of lung injury and act as signalling molecules to propagate an immune response, facilitating the recruitment of neutrophils to the injured alveolus [15]. Our findings in this cohort could be explained by the anti-platelet effect of aspirin, which prevents neutrophil recruitment and infiltration of the alveolar space. This hypothesis is supported by animal models of ARDS that show reductions in neutrophil migration to the alveolar space in mice treated with aspirin [29], suggesting that aspirin therapy is effective in reducing the severity of ARDS by modulating plateletneutrophil interactions.

Neutrophil recruitment to sites of lung injury may also be modulated through aspirin-triggered anti-inflammatory mediators. Aspirin acetylates cyclo-oxygenase-2, which results in the production of aspirin-triggered Resolvin D1 (AT-RvD1), an anti-inflammatory lipid mediator that can reduce neutrophil transendothelial migration in a peritonitis model [30]. In a murine model of ARDS, 
Table 2 Univariate analysis of ICU mortality

\begin{tabular}{|c|c|c|}
\hline \multirow[t]{2}{*}{ Predictor } & \multicolumn{2}{|l|}{ Univariate analysis } \\
\hline & Odds ratio $(95 \% \mathrm{Cl})$ & $P$-value \\
\hline Age & $1.03(1.00,1.05)^{*}$ & $0.01 *$ \\
\hline Male & $0.65(0.34,1.22)$ & 0.18 \\
\hline APACHE II score & $1.10(1.05,1.16)^{*}$ & $<0.001^{*}$ \\
\hline $\mathrm{PaO}_{2} / \mathrm{FiO}_{2}$ ratio & $0.97(0.93,1.00)^{*}$ & $0.06^{*}$ \\
\hline SOFA score & $1.35(1.21,1.50)^{*}$ & $<0.001^{*}$ \\
\hline Vasopressor use & $2.54(1.34,4.81)^{*}$ & $0.004^{*}$ \\
\hline Aspirin use (ever) & $0.75(0.37,1.53)^{*}$ & $0.43 *$ \\
\hline Smoking & $0.82(0.44,1.53)$ & 0.54 \\
\hline Coronary artery disease & $1.20(0.53,2.71)^{*}$ & $0.67^{*}$ \\
\hline Diabetes mellitus & $1.41(0.61,3.24)$ & 0.42 \\
\hline Pre-admission statin & $0.95(0.46,1.97)$ & 0.90 \\
\hline COPD & $0.83(0.33,2.07)$ & 0.69 \\
\hline Cerebrovascular disease & $0.88(0.23,3.40)$ & 0.86 \\
\hline Alcohol abuse history or cirrhosis & $1.30(0.67,2.54)$ & 0.44 \\
\hline Sepsis & $1.63(0.86,3.09)$ & 0.13 \\
\hline
\end{tabular}

*Values that were taken forward to multivariate analysis. APACHE II, acute physiology and chronic health evaluation; SOFA, sequential organ failure assessment; $\mathrm{PaO}_{2}$, arterial oxygen partial pressure; $\mathrm{FiO}_{2}$, fraction of inspired oxygen; COPD, chronic obstructive pulmonary disease.

AT-RVD1 treatment was shown to reduce neutrophil and macrophage recruitment to the site of lung injury, in addition to restoring the capillary-endothelial alveolar-epithelial barrier function. This suggests that aspirin therapy can decrease severity and augment resolution of ARDS [31].

Delayed neutrophil apoptosis is a prominent feature of ARDS [32], allowing inflammatory cells to remain within the alveolar space, prolonging the period of lung injury and hypoxia. Aspirin has previously been shown to preserve neutrophil apoptosis [17] and in the setting of ARDS, experimental evidence suggests that aspirin-triggered production of the anti-inflammatory lipid mediator 15-epilipoxin $\mathrm{A}_{4}$ restores neutrophil apoptosis and enhances the resolution of alveolar inflammation [33]. The effect of aspirin may be dose-dependent [34] and although our data

Table 3 Multivariate analysis of ICU mortality

\begin{tabular}{lll}
\hline Predictor & Odds ratio $(\mathbf{9 5 \%} \mathrm{Cl})$ & $\boldsymbol{P}$-value \\
\hline Aspirin use & $0.38(0.15,0.96)$ & $\mathbf{0 . 0 4}$ \\
Age & $1.02(1.00,1.05)$ & 0.10 \\
APACHE II score & $1.07(1.02,1.13)$ & $\mathbf{0 . 0 1 *}$ \\
Coronary artery disease & $1.16(0.43,3.10)$ & 0.77 \\
$\mathrm{PaO}_{2} / \mathrm{FiO}_{2}$ ratio & $0.97(0.93,1.00)$ & 0.08 \\
Vasopressor use & $2.09(1.05,4.18)$ & $\mathbf{0 . 0 4}$
\end{tabular}

*Values that were deemed significant. APACHE II score on admission is presented as a continuous variable. APACHE II, acute physiology and chronic health evaluation; $\mathrm{PaO}_{2}$, arterial oxygen partial pressure; $\mathrm{FiO}_{2}$, fraction of inspired oxygen.
Table 4 Multivariate model for analysis of ICU mortality including patients with combined aspirin and statin exposure

\begin{tabular}{lll}
\hline Predictor & Odds ratio $(\mathbf{9 5} \% \mathrm{Cl})$ & $\boldsymbol{P}$-value \\
\hline Aspirin and statin exposure & $0.68(0.26,1.80)$ & 0.44 \\
Age & $1.01(0.99,1.04)$ & 0.21 \\
APACHE II score & $1.07(1.02,1.13)$ & $\mathbf{0 . 0 1 *}$ \\
Coronary artery disease & $1.01(0.39,2.63)$ & 0.99 \\
$\mathrm{PaO}_{2} / \mathrm{FiO}_{2}$ ratio & $0.97(0.94,1.01)$ & 0.15 \\
Vasopressor use & $2.06(1.04,4.08)$ & $\mathbf{0 . 0 4}$ \\
\hline
\end{tabular}

*Values that were deemed significant. APACHE II score on admission is presented as a continuous variable.

support the role of low-dose therapy, we require future clinical trials to investigate the most appropriate dose for aspirin in ARDS.

Prior observational studies involving aspirin and ARDS suggest an association between aspirin exposure and prevention of ARDS in at-risk groups [20-22], but as yet there is no evidence about aspirin use as a therapy for ARDS. In sepsis, the most common precipitant of ARDS, observational studies have shown reduced mortality when aspirin is administered during the course of illness $[24,25]$. In addition, there are clinical implications from our audit. In our cohort, $63 \%$ of pre-hospital aspirin users had their anti-platelet therapy discontinued at ICU admission, often without documented justification. Previous observational studies have shown reduced ICU mortality in patients exposed to aspirin prior to ICU admission [35], and the addition of our data suggests that consideration should be given to continuing aspirin at ICU admission provided no contraindications exist [36]. Pre-admission statin prescription was higher in the aspirin cohort. The combined effect of aspirin and statin treatment has previously been associated with a reduced incidence of ARDS [19], however in our cohort there was no association with an ICU survival advantage, suggesting that there is limited therapeutic benefit of combined treatment in ARDS. This finding adds to recent clinical trials suggesting that there is a limited role for statin therapy in ARDS [37,38].

Of the different subgroups of patients treated, those who were prescribed aspirin within ICU showed the greatest survival benefit although this did not reach statistical significance, which may have been related to the small patient number. We are cautious in our interpretation of this, and believe that further clinical trials are required to assess the benefit of aspirin as a treatment for ARDS.

Our patients were prospectively identified as part of ongoing screening for recruitment to clinical trials within our ICU, and we believe this identification process to be a strength that supports the reliability of our findings. We found APACHE II score and vasopressor use to be 


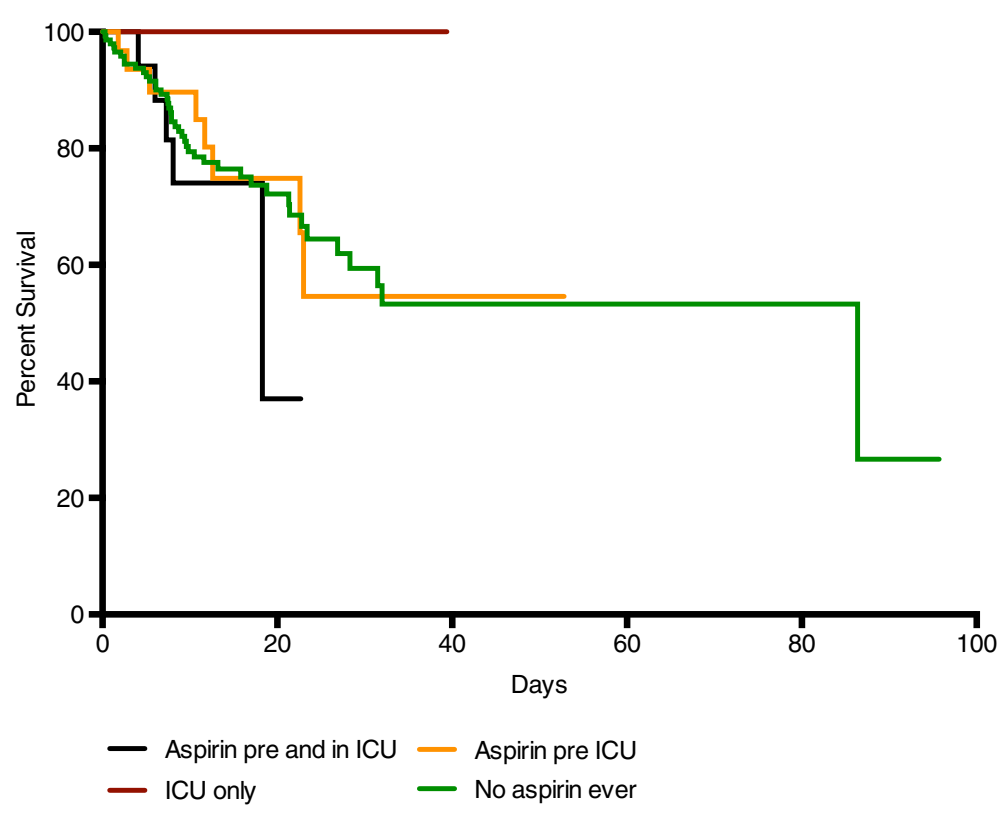

Figure 1 ICU survival in different aspirin exposure groups. Kaplan-Meier graph showing survival status for patients in the four different subgroups of aspirin treatment. There was no significant difference in survival amongst the groups (log rank test, $P=0.48$ ).

associated with increased ICU mortality from ARDS, and this finding confirms previous studies that have shown these as independent predictors of ICU mortality [39], supporting that this cohort is representative.

The limitations of our findings are in keeping those of observational studies. The aspirin cohort had higher rates of associated co-morbidity, and it could be that aspirin usage is a surrogate for an unknown confounding factor. We opted to combine patients who received aspirin pre-admission and during admission into a single cohort because of the prolonged inhibition of thromboxane A2 for up to 10 days [28]. However many of our patients did not receive aspirin within ICU. In addition, adverse events associated with the continuation of aspirin in ICU were not consistently recorded. Therefore, it is feasible that the efficacy and safety profile could be different when aspirin is used specifically as a treatment for ARDS in ICU. Although prior observational data suggest benefit in continuing aspirin in patients with a high risk of bleeding [35], it is important that the potential risks associated with aspirin in this patient population are clarified in future clinical trials of aspirin as a treatment for ARDS. These findings may have been confounded by the so-called healthy user effect [40], whereby the more unwell patients have their medications, including aspirin, discontinued. Although unable to control for this in our cohort, we believe the finding that both APACHE II and SOFA scores were similar between the groups suggests that this effect has not had a significant impact upon our findings. The use of ICU mortality as the primary outcome measure is a potential limitation, however we believe it reflects the outcome specifically from ARDS, whilst the cause of hospital mortality may be unrelated to the development of ARDS. However the lack of an association with aspirin usage and hospital mortality may indicate the association with aspirin and clinical outcomes is limited and therefore further research is required to confirm these findings. Finally, smoking and alcohol consumption histories were obtained from review of the medical chart. We acknowledge that this is a potential limitation in the assessment of these factors within our cohort and may not fully represent the confounding effects of smoking and alcohol [41].

At present our findings are hypothesis-generating, and support the need for clinical trials to investigate aspirin as a therapy for ARDS. In addition to trials investigating aspirin as a preventative treatment for ARDS [42], our group is currently investigating aspirin in a model of ARDS induced by inhaled lipopolysaccharide (LPS) in healthy volunteers (ARENA, NCT01659307) and are planning a phase-2 study of aspirin in patients with ARDS (STAR, NCT02326350). Both of these trials will better inform clinicians as to the clinical potential of aspirin as a therapy for ARDS.

\section{Conclusion}

In summary, our audit demonstrates an association between aspirin use and reduced ICU mortality in patients with ARDS. Early-phase clinical trials investigating the potential for aspirin as a therapy for ARDS are needed. 


\section{Key messages}

- In this audit aspirin therapy is associated with reduced risk of ICU mortality in patients with ARDS.

- APACHE II score and vasopressor use correlate with ICU mortality.

- There is a need for clinical trials investigating the role for aspirin in ARDS.

\section{Abbreviations}

APACHE: acute physiology and chronic health evaluation; ARDS: acute respiratory distress syndrome; AT-RvD1: aspirin-triggered Resolvin D1; $\mathrm{FiO}_{2}$ : fraction of inspired oxygen; $\mathrm{Cl}$ : Confidence Intervals; HR: hazard ratio; LPS: Lipopolysaccharide; OR: odds ratio; $\mathrm{PaO}_{2}$ : partial pressure of oxygen; SIRS: systemic inflammatory response syndrome; SOFA: sequential organ failure assessment.

\section{Competing interests}

DFM has performed paid consultancy work and has been a member of advisory boards on ARDS for Glaxo-SmithKline. This author's institution has been paid for the author to undertake bronchosocopy as part of a clinical trial funded by Glaxo-SmithKline. DFM has a patent submitted for a novel treatment (unrelated to aspirin) for ARDS, and is the chief investigator of a multi-centre study investigating simvastatin as a therapy for ARDS (funded by the National Institute for Health and Research), is the chief investigator of a single centre study investigating aspirin in ARDS (funded by the Northern Ireland Research and Development Office) and is chief investigator of a single centre study investigating aspirin in a model of ARDS ((ARENA, NCT01659307) funded by the UK Intensive Care Society). $\mathrm{CMO}$ is a co-investigator of a single centre study investigating Aspirin in ARDS (funded by the Northern Ireland Research and Development Office) and co-investigator of a single centre study investigating aspirin in a model of ARDS ((ARENA, NCT01659307) funded by the UK Intensive Care Society). All other authors declare that they have no competing interests.

\section{Authors' contributions}

AJB, UIH, JJM, CMO and DFM conceived and designed the project. AJB, LJM, UIH, LM, GW and LJMC performed data collection. SDG performed the statistical analysis. AJB, CMO and DFM were involved in the interpretation of the data. AJB and SDG drafted the first version of the manuscript and all authors critically revised the manuscript and approved the final version.

\section{Authors' information}

AJB: Core Medical Trainee, Regional Intensive Care Unit, Royal Victoria Hospital, Belfast Northern Ireland Deanery. SDG: Senior Statistician Epidemiology ASL TO3, Via Sabaudia, 164, 10095, Grugliasco (TO), Italy. UIH: Clinical Research Fellow Centre for Infection and Immunity, Queen's University Belfast. LJM: Consultant Anaesthetist and Intensivist. Royal Victoria Hospital, Belfast. LM: Research Physician Assistant Regional Intensive Care Unit, Royal Victoria Hospital, Belfast. GW: Research Assistant Regional Intensive Care Unit, Royal Victoria Hospital, Belfast. LJMC: Doctoral Fellow, Centre for Infection and Immunity, Queen's University Belfast. JJM: Consultant Anaesthetist and Intensivist Royal Victoria Hospital, Belfast. CMO: Consultant Respiratory Physician/Senior Lecturer in Respiratory Medicine Regional Respiratory Centre/Centre for Infection and Immunity, Belfast City Hospital/Queen's University Belfast. DFM: Consultant Intensivist/Professor of Intensive Care Medicine Regional Intensive Care Unit/Centre for Infection and Immunity, Royal Victoria Hospital, Belfast/Queen's University Belfast.

\section{Author details}

${ }^{1}$ Centre for Infection and Immunity, Health Sciences Building, Queen's University Belfast, 97 Lisburn Road, Belfast, UK. ${ }^{2}$ Regional Intensive Care Unit, Royal Victoria Hospital, Grosvenor Road, Belfast, UK. ${ }^{3}$ Epidemiology ASL TO3, Via Sabaudia, 164, Grugliasco, TO 10095, Italy.

Received: 22 July 2014 Accepted: 2 March 2015

Published online: 23 March 2015

\section{References}

1. Rubenfeld GD, Caldwell E, Peabody E, Weaver J, Martin DP, Neff M, et al. Incidence and outcomes of acute lung injury. N Engl J Med. 2005;353:1685-93.

2. Herridge MS, Cheung AM, Tansey CM, Matte-Martyn A, Diaz-Granados N, Al-Saidi $F$, et al. One-year outcomes in survivors of the acute respiratory distress syndrome. N Engl J Med. 2003;348:683-93.

3. Herridge MS, Tansey CM, Matté A, Tomlinson G, Diaz-Granados N, Cooper A, et al. Functional disability 5 years after acute respiratory distress syndrome. N Engl J Med. 2011;364:1293-304.

4. Perkins GD, Nathani N, McAuley DF, Gao F, Thickett DR. In vitro and in vivo effects of salbutamol on neutrophil function in acute lung injury. Thorax. 2007;62:36-42.

5. Abraham E. Neutrophils and acute lung injury. Crit Care Med. 2003;31:S195-9.

6. Frank JA, Wray CM, McAuley DF, Schwendener R, Matthay MA. Alveolar macrophages contribute to alveolar barrier dysfunction in ventilator-induced lung injury. Am J Physiol - Lung Cell Mol Physiol. 2006;291:L1191-8.

7. Ware LB, Matthay MA. The acute respiratory distress syndrome. N Engl J Med. 2000:342:1334-49.

8. Boyle AJ, Sweeney RM, McAuley DF. Pharmacological treatments in ARDS; a state-of-the-art update. BMC Med. 2013;11:166.

9. Boyle AJ, McNamee JJ, McAuley DF. Biological therapies in the acute respiratory distress syndrome. Expert Opin Biol Ther. 2014;14:969-81.

10. The Acute Respiratory Distress Syndrome Network. Ventilation with lower tidal volumes as compared with traditional tidal volumes for acute lung injury and the acute respiratory distress syndrome. N Engl J Med. 2000;342:1301-8

11. Guérin C, Reignier J, Richard J-C, Beuret P, Gacouin A, Boulain T, et al. Prone positioning in severe acute respiratory distress syndrome. N Engl J Med. 2013;368:2159-68.

12. Papazian L, Forel J-M, Gacouin A, Penot-Ragon C, Perrin G, Loundou A, et al. Neuromuscular blockers in early acute respiratory distress syndrome. N Engl J Med. 2010;363:1107-16.

13. Peek GJ, Mugford M, Tiruvoipati R, Wilson A, Allen E, Thalanany MM, et al. Efficacy and economic assessment of conventional ventilatory support versus extracorporeal membrane oxygenation for severe adult respiratory failure (CESAR): a multicentre randomised controlled trial. The Lancet. 2009;374:1351-63.

14. Fitzgerald M, Millar J, Blackwood B, Davies A, Brett SJ, McAuley DF, et al. Extracorporeal carbon dioxide removal for patients with acute respiratory failure secondary to the acute respiratory distress syndrome: a systematic review. Crit Care. 2014;18:222.

15. Zarbock A, Singbartl K, Ley K. Complete reversal of acid-induced acute lung injury by blocking of platelet-neutrophil aggregation. J Clin Invest. 2006:116:3211-9.

16. Looney MR, Nguyen JX, Hu Y, Van Ziffle JA, Lowell CA, Matthay MA. Platelet depletion and aspirin treatment protect mice in a two-event model of transfusion-related acute lung injury. J Clin Invest. 2009;119:3450-61.

17. Bates JJ, Watson RWG, Glynn CM, O'Neill AJ, Fitzpatrick JM, Buggy DJ. Aspirin preserves neutrophil apoptosis after cardiopulmonary bypass. Shock Augusta Ga. 2004;21:495-9.

18. Kor DJ, Erlich J, Gong MN, Malinchoc M, Carter RE, Gajic O, et al. Association of prehospitalization aspirin therapy and acute lung injury: results of a multicenter international observational study of at-risk patients. Crit Care Med. 2011;39:2393-400.

19. O'Neal HR, Koyama T, Koehler EAS, Siew E, Curtis BR, Fremont RD, et al. Prehospital statin and aspirin use and the prevalence of severe sepsis and ALI/ARDS. Crit Care Med. 2011;39:1343-50.

20. Harr JN, Moore EE, Johnson J, Chin TL, Wohlauer MV, Maier R, et al. Antiplatelet therapy is associated with decreased transfusion-associated risk of lung dysfunction, multiple organ failure, and mortality in trauma patients. Crit Care Med. 2013:41:399-404.

21. Erlich JM, Talmor DS, Cartin-Ceba R, Gajic O, Kor DJ. Prehospitalization antiplatelet therapy is associated with a reduced incidence of acute lung injury: a population-based cohort study. Chest. 2011;139:289-95.

22. Ahmed AHM, Litell JMD, Malinchoc MM, Kashyap RM, Schiller HJ, Pannu SRM, et al. The role of potentially preventable sospital exposures in the development of acute respiratory distress syndrome: A population-based study. Crit Care Med. 2014:42:31-9.

23. Winning J, Reichel J, Eisenhut Y, Hamacher J, Kohl M, Deigner HP, et al. Anti-platelet drugs and outcome in severe infection: Clinical impact and underlying mechanisms. Platelets. 2009;20:50-7. 
24. Eisen DP, Reid D, McBryde ES. Acetyl salicylic acid usage and mortality in critically ill patients with the systemic inflammatory response syndrome and sepsis. Crit Care Med. 2012;40:1761-7.

25. Sossdorf M, Otto GP, Boettel J, Winning J, Lösche W. Benefit of low-dose aspirin and non-steroidal anti-inflammatory drugs in septic patients. Crit Care. 2013;17:402.

26. Bernard GR, Artigas A, Brigham KL, Carlet J, Falke K, Hudson L, et al. The American-European Consensus Conference on ARDS. Definitions, mechanisms, relevant outcomes, and clinical trial coordination. Am J Respir Crit Care Med. 1994;149:818-24.

27. The ARDS Definition Task Force. Acute respiratory distress syndrome: The Berlin definition. JAMA. 2012;307:2526-33.

28. Wood AJJ, Patrono C. Aspirin as an antiplatelet drug. N Engl J Med. 1994;330:1287-94.

29. Tuinman PR, Müller MC, Jongsma G, Hegeman MA, Juffermans NP. High-dose acetylsalicylic acid is superior to low-dose as well as to clopidogrel in preventing lipopolysaccharide-induced lung injury in mice. Shock Augusta Ga. 2013;40:334-8.

30. Sun Y-P, Oh SF, Uddin J, Yang R, Gotlinger K, Campbell E, et al. Resolvin D1 and its aspirin-triggered 17R epimer stereochemical assignments, anti-inflammatory properties, and enzymatic inactivation. J Biol Chem. 2007;282:9323-34

31. Eickmeier O, Seki H, Haworth O, Hilberath J, Gao F, Uddin M, et al. Aspirin-Triggered-Resolvin D1 reduces mucosal inflammation and promotes resolution in a murine model of acute lung injury. Mucosal Immunol. 2013;6:256-66.

32. Matute-Bello G, Liles WC, Radella F, Steinberg KP, Ruzinski JT, Jonas M, et al. Neutrophil apoptosis in the acute respiratory distress syndrome. Am J Respir Crit Care Med. 1997;156:1969-77.

33. El Kebir D, József $L$, Pan W, Wang L, Petasis NA, Serhan CN, et al. 15-epi-lipoxin A4 inhibits myeloperoxidase signaling and enhances resolution of acute lung injury. Am J Respir Crit Care Med. 2009;180:311-9.

34. Morris T, Stables M, Hobbs A, de Souza P, Colville-Nash P, Warner T, et al. Effects of low-dose aspirin on acute inflammatory responses in humans. J Immunol. 2009;183:2089-96.

35. Winning J, Neumann J, Kohl M, Claus RA, Reinhart K, Bauer M, et al. Antiplatelet drugs and outcome in mixed admissions to an intensive care unit. Crit Care Med. 2010;38:32-7.

36. Mahajan $\mathrm{R}$, Heptinstall S. Should critical care patients receive or remain on treatment with antiplatelet drugs? Crit Care Med. 2010;38:298-300

37. McAuley DF, Laffey JG, O'Kane CM, Perkins GD, Mullan B, Trinder TJ, et al Simvastatin in the acute respiratory distress syndrome. N Engl J Med. 2014;371:1695-703.

38. The National Heart, Lung, and Blood Institute ARDS Clinical Trials Network. Rosuvastatin for sepsis-associated acute respiratory distress syndrome. N Engl J Med. 2014;370:2191-200.

39. Rocker G, Cook D, Sjokvist P, Weaver B, Finfer S, McDonald E, et al. Clinician predictions of intensive care unit mortality. Crit Care Med. 2004:32:1149-54.

40. The Irish Critical Care Trials Group. Acute lung injury and the acute respiratory distress syndrome in Ireland: a prospective audit of epidemiology and management. Crit Care. 2008;12:R30.

41. Hsieh SJ, Zhuo H, Benowitz NL, Thompson BT, Liu KD, Matthay MA, et al. Prevalence and impact of active and passive cigarette smoking in acute respiratory distress syndrome. Crit Care Med. 2014;42:2058-68.

42. Kor DJ, Talmor DS, Banner-Goodspeed VM, Carter RE, Hinds R, Park PK, et al. Lung Injury Prevention with Aspirin (LIPS-A): a protocol for a multicentre randomised clinical trial in medical patients at high risk of acute lung injury. BMJ Open. 2012;2.

\section{Submit your next manuscript to BioMed Central and take full advantage of:}

- Convenient online submission

- Thorough peer review

- No space constraints or color figure charges

- Immediate publication on acceptance

- Inclusion in PubMed, CAS, Scopus and Google Scholar

- Research which is freely available for redistribution 